\title{
Comparison of the clinical performance of i-gel, LMA Supreme and LMA ProSeal in elective surgery
}

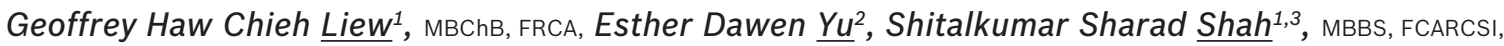 \\ Harikrishnan Kothandan ${ }^{1,3}$, MBBS, FANZCA
}

\begin{abstract}
INTRODUCTION The LMA Supreme ${ }^{T M}$, i-gel® and LMA ProSeal ${ }^{T M}$ are second-generation supraglottic airway devices. We tested the hypothesis that these devices differ in performance when used for spontaneous ventilation during anaesthesia. METHODS 150 patients who underwent general anaesthesia for elective surgery were randomly allocated into three groups. Data was collected on oropharyngeal leak pressures, ease and duration of device insertion, ease of gastric tube insertion, and airway safety.

RESULTS Leak pressure, our primary outcome measure, was found to be higher for the i-gel than the Supreme and ProSeal (mean \pm standard error of the mean: $27.31 \pm 0.92 \mathrm{cmH}_{2} \mathrm{O}, 23.60 \pm 0.70 \mathrm{cmH}_{2} \mathrm{O}$ and $24.44 \pm 0.70 \mathrm{cmH}_{2} \mathrm{O}$, respectively; $p=0.003$ ). Devices were inserted on the first attempt for $90 \%, 82 \%$ and $72 \%$ of patients in the i-gel, Supreme and ProSeal groups, respectively $(p=0.105)$; mean device placement times were 23.58 seconds, 25.10 seconds and 26.34 seconds, respectively $(p=0.477)$. Gastric tubes were inserted on the first attempt in $100 \%$ of patients in the Supreme group, and $94 \%$ of patients in the i-gel and ProSeal groups $(p=0.100)$. There was blood staining on removal in $9(18 \%)$ patients in each of the Supreme and ProSeal groups, with none in the i-gel group $(p=0.007)$. The incidence of postoperative sore throat, dysphagia and hoarseness was lowest for the i-gel.

CONCLUSION The three devices were comparable in terms of ease and duration of placement, but the i-gel had higher initial oropharyngeal leak pressure and lower airway morbidity compared with the ProSeal and Supreme.
\end{abstract}

Keywords: airway, i-gel, LMA, ProSeal, Supreme

\section{INTRODUCTION}

The classic laryngeal mask airway (LMA), introduced by Brain in 1988, revolutionised the practice of airway management and is now routinely utilised in clinical anaesthesia. ${ }^{(1)}$ Nevertheless, there are still limitations associated with the classic LMA, such as controlled ventilation being relatively contraindicated (due to its moderate oropharyngeal seal) and its unsuitability for patients at risk of aspiration. ${ }^{(2)}$ Second-generation supraglottic airway devices (SADs) were designed to address these issues. The newer SADs have additional safety features that enhance the oesophageal and pharyngeal seals; the risk of aspiration is also minimised with the introduction of the gastric channel, which enables gastric suctioning, venting and passage of a nasogastric tube.

Second-generation SADs that are commonly used are the LMA ProSealTM (Teleflex Medical Europe Ltd, County Westmeath, Ireland), LMA Supreme ${ }^{\mathrm{TM}}$ (LMA-S ${ }^{\mathrm{TM}}$; The Laryngeal Mask Company Pte Ltd, Singapore) and i-gel $®$ (Intersurgical Ltd, Wokingham, UK). The ProSeal is a reusable device made of silicone with an inbuilt gastric port, an inflatable posterior pharyngeal cuff for better airway seal and a rigid bite block. The Supreme, introduced commercially in 2007, is a singleuse SAD made of polyvinyl chloride with a gastric drain tube, large inflatable plastic cuff and preformed semi-rigid tube. The i-gel, also clinically introduced in 2007, is a single-use device comprising a soft gel-like cuffless mask, a narrow-bore gastric drain tube and an integral bite block. Numerous previous studies of these airway devices have demonstrated their easy, reliable insertion and low morbidity rate. ${ }^{(3-7)}$

However, comparative studies involving all three aforementioned airway devices are lacking. One study testing the three devices used the laryngoscope-guided and gastric tubeguided methods of insertion, while another was conducted on paralysed, ventilated patients undergoing laparoscopic surgery. ${ }^{(8,9)}$ In contrast, our randomised clinical study employed the standard, manufacturer-recommended insertion techniques. We compared the clinical efficacy of all three devices in non-paralysed patients undergoing elective surgery, taking oropharyngeal leak pressure as the primary outcome measure. In addition, we tested secondary variables such as the ease and speed of SAD insertion, ease of gastric tube passage and perioperative airway complications.

\section{METHODS}

We recruited a total of 150 patients who were (a) aged 21-80 years; (b) of American Society of Anesthesiologists Grades I-III; and (c) due to undergo elective superficial or peripheral surgery in our tertiary hospital in the supine position, with an operation duration of more than 30 minutes. Exclusion criteria were body mass index $>35 \mathrm{~kg} / \mathrm{m}^{2}$, history of known or predicted difficult airway, and known risk of aspiration. The study was approved by the SingHealth Centralised Institutional Review Board (reference: 2014/393/D) and written informed consent was obtained from all patients.

${ }^{1}$ Department of Anaesthesiology, Singapore General Hospital, 2Duke-NUS Graduate Medical School, ${ }^{3}$ National Heart Centre, Singapore

Correspondence: Dr Harikrishnan Kothandan, Consultant Anaesthesiologist, Department of Anaesthesiology, Singapore General Hospital, Outram Road, Singapore 169608. harikrishnan.kothandan@sgh.com.sg 
Patients were randomly allocated into three groups: Supreme, ProSeal and i-gel. Randomisation was performed using a random number table (http://www.randomization.com/). The size of the SAD was selected based on the patient's weight, as per the manufacturers' size recommendations. For the Supreme and ProSeal groups, sizes 3, 4 and 5 were used for weights of $30-50 \mathrm{~kg}, 50-70 \mathrm{~kg}$ and $>70 \mathrm{~kg}$, respectively. For the i-gel group, the recommendation was for sizes 3,4 and 5 to be used for weights of $30-60 \mathrm{~kg}, 50-90 \mathrm{~kg}$ and $>90 \mathrm{~kg}$, respectively. However, since there was an overlap at the weights of 50-60 kg for i-gel sizes 3 and 4 , the weight limits were modified to size 3 for $30-54.9 \mathrm{~kg}$ and size 4 for 55-90 kg for the purpose of this study.

Standard monitoring (i.e. noninvasive blood pressure taking, pulse oximetry and electrocardiography) was applied before anaesthetic induction. After three minutes of preoxygenation, anaesthetic induction was initiated with intravenous fentanyl $1-2 \mu \mathrm{g} / \mathrm{kg}$ and intravenous propofol $2-3 \mathrm{mg} / \mathrm{kg}$. The lungs were then manually ventilated via face mask and oral airway if required, using sevoflurane $2 \%$ in oxygen $100 \%$, until there was a lack of response to jaw thrust and the jaw was adequately relaxed; additional intravenous boluses of propofol up to $0.5 \mathrm{mg} / \mathrm{kg}$ were administered if required. After post-induction apnoea occurred, the chosen SADs were lubricated and inserted according to the manufacturers' recommendations. The Supreme and i-gel SADs were inserted using the rotational technique, whereas the ProSeal SAD was inserted using the index finger. The cuffs of the inflatable SADs, i.e. Supreme and ProSeal, were inflated with air to $60 \mathrm{cmH}_{2} \mathrm{O}$. Maintenance of anaesthesia was achieved using a mixture of oxygen and air with 1-1.5 minimum alveolar concentration sevoflurane. The patients were either spontaneously breathing or were temporarily placed on the ventilator with tidal volume set at $6 \mathrm{~mL} / \mathrm{kg}$ and respiratory rate at 10-14 breaths/minute until spontaneous ventilation occurred.

All the SAD insertions were performed by the experienced co-investigators. The speed of SAD insertion was obtained by recording the time from which the SAD was picked up to the time it was correctly placed with two effective end-tidal capnography waveforms. The ease of insertion was classified based on insertion attempts ( 1 = first attempt, 2 = second attempt, 3 = third attempt, $4=$ intubated and $5=$ size change required). Following SAD insertion, a lubricated gastric tube was inserted though the gastric channel (size 12 FG for i-gel, and size 14 FG for both ProSeal and Supreme). Ease of gastric tube insertion was graded on a three-point scale ( 1 = first attempt, 2 = second attempt and 3 = impossible). If the gastric tube was successfully inserted, gastric content would be aspirated and the amount was recorded. Immediately after correct SAD placement, the oropharyngeal leak pressure was measured once. This was done following full closure of the adjustable pressure valve with a fresh gas flow of $3 \mathrm{~L} / \mathrm{min}$. The leak pressure was measured using auscultation, i.e. airway pressure when there is a detectable leak sound in the throat or mouth; the maximum pressure allowed was $40 \mathrm{cmH}_{2} \mathrm{O}$. Patients who were spontaneously breathing during the leak pressure tests were excluded from the study.

Perioperative adverse events and complications (i.e. dental, lip or tongue injury; oxygen desaturation; ventilation problems; laryngospasm; and aspiration/regurgitation) were also documented. At the end of the procedure, the SAD was removed upon eye opening by the patient. The airway device was inspected for any evidence of blood or gastric content. The patient was then asked, one hour later in the recovery room and the following day, to report any sore throat, hoarseness of voice or dysphagia. These symptoms were graded as mild, moderate or severe.

The primary comparison parameter was oropharyngeal leak pressure. Sample size was estimated from a previous similar study that detected oropharyngeal leak pressure differences among the i-gel (mean \pm standard deviation $[\mathrm{SD}]=28.3 \pm 2.4 \mathrm{cmH}_{2} \mathrm{O}$ ), ProSeal (mean $\pm \mathrm{SD}=25.0 \pm 2.3 \mathrm{cmH}_{2} \mathrm{O}$ ) and Supreme (mean $\pm \mathrm{SD}=25.0 \pm 1.9 \mathrm{cmH}_{2} \mathrm{O}$ ) SADs, $\mathrm{p}<0.001$; that study used a sample size of 35 patients in each group at 30 minutes upon insertion. ${ }^{(9)}$ Therefore, we recruited 50 patients for each group and expected a similar effect size at the 0.05 level of significance with a power of $100 \%$. Statistical analysis was performed using IBM SPSS Statistics version 20.0 for Macintosh (IBM Corp, Armonk, NY, USA). Continuous variables were reported as mean \pm standard error of the mean (SEM) and categorical variables were reported as number (percentage). Normality for continuous variables in groups was determined by Shapiro-Wilk test. One-way analysis of variance, Dunnett's test and Kruskal-Wallis analysis of variance with post hoc analysis were used for comparison of continuous variables among groups. Pearson's chi-square test was used to compare categorical variables among studied groups. A value of $\mathrm{p}<0.05$ was deemed statistically significant.

\section{RESULTS}

A total of 150 patients were included in the study and no patients dropped out. Their demographic data and airway characteristics, as well as surgery information, are summarised in Table I. Most parameters were similarly distributed among the three study groups (except for dentition), which showed successful randomisation. Oropharyngeal leak pressure differences were detected among the i-gel (mean $\pm \mathrm{SEM}=27.31 \pm 0.92 \mathrm{cmH}_{2} \mathrm{O}$ ), ProSeal (mean \pm SEM $=24.44 \pm 0.70 \mathrm{cmH}_{2} \mathrm{O}$ ) and Supreme (mean $\left.\pm \mathrm{SEM}=23.60 \pm 0.70 \mathrm{cmH}_{2} \mathrm{O}\right)$ SADs $(\mathrm{p}<0.003)$. Post hoc analysis showed that the mean oropharyngeal leak pressure of the i-gel SAD was significantly higher than that of the Supreme (mean difference \pm SEM $=3.71 \pm 1.11 \mathrm{cmH}_{2} \mathrm{O} ; \mathrm{p}=0.002$ ) and ProSeal (mean difference $\pm \mathrm{SEM}=2.87 \pm 1.11 \mathrm{cmH}_{2} \mathrm{O} ; \mathrm{p}=0.020$ ) SADs (Table II).

Most airway insertion characteristics and ventilatory parameters were similar among the three groups (Table II); however the SAD size distribution was one point smaller in the i-gel group as compared with those of the other groups $(p<0.001)$. The success rates of the first insertion attempt with the i-gel, Supreme and ProSeal SADs were $90 \%, 82 \%$ and $72 \%$, respectively. SAD placement time was slightly shorter in the i-gel group compared to the ProSeal and Supreme groups, but the p-value was not significant due to limited power. The success rates of gastric tube insertion on first attempt with the i-gel, Supreme and ProSeal SADs were 94\%, $100 \%$ and $94 \%$, respectively. Gastric tube aspirate amounts were different among the groups 
Table l. Characteristics of patients and surgery $(n=150)$.

\begin{tabular}{|c|c|c|c|c|}
\hline \multirow[t]{2}{*}{ Parameter } & \multicolumn{3}{|c|}{ No. (\%) } & \multirow[t]{2}{*}{ p-value } \\
\hline & i-gel $(n=50)$ & Supreme $(n=50)$ & ProSeal $(n=50)$ & \\
\hline Age* (yr) $^{*}$ & $51.36 \pm 1.98$ & $48.40 \pm 2.33$ & $53.92 \pm 2.25$ & 0.207 \\
\hline Gender & & & & 0.482 \\
\hline Male & $25(50)$ & $31(62)$ & $28(56)$ & \\
\hline Female & $25(50)$ & $19(38)$ & $22(44)$ & \\
\hline Height* $^{*}(\mathrm{~cm})$ & $161.68 \pm 1.09$ & $164.26 \pm 1.39$ & $161.42 \pm 1.19$ & 0.199 \\
\hline Weight* (kg) & $63.00 \pm 1.86$ & $66.78 \pm 2.17$ & $65.60 \pm 1.46$ & 0.341 \\
\hline Body mass index* $\left(\mathrm{kg} / \mathrm{cm}^{2}\right)$ & $23.72 \pm 0.58$ & $24.56 \pm 0.61$ & $24.72 \pm 0.44$ & 0.387 \\
\hline ASA class & & & & 0.829 \\
\hline I & $19(38)$ & $20(40)$ & $17(34)$ & \\
\hline II & $27(54)$ & $23(46)$ & $27(54)$ & \\
\hline III & $4(8)$ & $7(14)$ & $6(12)$ & \\
\hline Dentition & & & & 0.004 \\
\hline Own & $35(70)$ & $33(66)$ & $26(52)$ & \\
\hline Full dentures & $4(8)$ & $8(16)$ & $8(16)$ & \\
\hline Partial dentures & $8(16)$ & $9(18)$ & $14(28)$ & \\
\hline Crown & $3(6)$ & 0 & $2(4)$ & \\
\hline Mallampati class & & & & 0.342 \\
\hline 1 & $19(38)$ & $23(46)$ & $18(36)$ & \\
\hline 2 & $28(56)$ & $27(54)$ & $31(62)$ & \\
\hline 3 & $3(6)$ & 0 & $1(2)$ & \\
\hline Type of surgery & & & & 0.728 \\
\hline General & $11(22)$ & $11(22)$ & $11(22)$ & \\
\hline Gynaecology & $7(14)$ & $6(12)$ & $3(6)$ & \\
\hline Orthopaedic & $16(32)$ & $13(26)$ & $9(18)$ & \\
\hline Plastic & $1(2)$ & $2(4)$ & $2(4)$ & \\
\hline Urology & $14(28)$ & $16(32)$ & $22(44)$ & \\
\hline Vascular & $1(2)$ & $2(4)$ & $3(6)$ & \\
\hline Duration of anaesthesia $^{+}(\mathrm{min})$ & $70.28(30-190)$ & $72.96(30-180)$ & $67.96(32-165)$ & 0.697 \\
\hline
\end{tabular}

* Data presented as mean \pm standard error of the mean. ${ }^{\dagger}$ Data presented as mean (range). ASA: American Society of Anesthesiologists

$(p=0.035)$, with post hoc analysis showing that the i-gel group had slightly higher volumes of gastric aspirates compared with the Supreme group $(p=0.044)$. However, the difference with the ProSeal group was not significant $(p=0.142)$ (Table II).

Intraoperative and postoperative complications among the groups are compared in Table III. Intraoperatively, there was one episode of laryngospasm in the Supreme group and one case of airway obstruction in the ProSeal group, which was resolved after repositioning. Upon device removal, visible blood contamination in the SAD was noticed in 9 (18\%) patients in each of the ProSeal and Supreme groups, although none was observed in the i-gel group $(p=0.007)$. Gastric content stains were found in the i-gel and Supreme SADs on removal (one case each). Surprisingly, there were $3(6 \%)$ cases of significant air leak at induction in the i-gel group, but none in the other groups. This seemed to correlate with the size of the SAD, as size 3 SADs were used in all three cases; two patients had tracheal intubations, and another patient was changed to ProSeal and subsequently excluded from further analyses. In the one-hour postoperative evaluation, dysphagia was not observed in the i-gel group but was present in $7(14 \%)$ and $3(6 \%)$ patients in the Supreme and ProSeal groups, respectively; there were fewer complaints of sore throat in the i-gel group $(4 \%, \mathrm{n}=2)$ compared with the Supreme $(28 \%, \mathrm{n}=14)$ and ProSeal $(30 \%$, $\mathrm{n}=15$ ) groups. These differences in dysphagia and sore throat were statistically significant ( $p=0.020$ and 0.002 , respectively). Hoarseness of voice was not observed in the i-gel group, but was present in the Supreme $(2 \%, \mathrm{n}=1)$ and ProSeal $(4 \%, \mathrm{n}=2)$ groups, although the difference was not statistically significant $(p=0.367)$. At follow-up on the next day, $2(4 \%)$ cases of dysphagia and $8(16 \%)$ cases of sore throat were found to be persistent in the Supreme group, while 1 ( $2 \%$ ) case of dysphagia, $1(2 \%)$ case of hoarseness and $4(8 \%)$ cases of sore throat were persistent in the ProSeal group. Only 2 (4\%) cases of sore throat were observed in the i-gel group. Nevertheless, these values were not significant. Most of the symptoms were reported as mild on the severity index.

\section{DISCUSSION}

In the present study, we found that the mean oropharyngeal leak pressure following induction was higher in the i-gel group (27.31 $\mathrm{cmH}_{2} \mathrm{O}$ ) than the Supreme (23.60 $\mathrm{cmH}_{2} \mathrm{O}$ ) and ProSeal (24.44 $\mathrm{cmH}_{2} \mathrm{O}$ ) groups. In general, it is thought that higher oropharyngeal leak pressures in SADs allow the use 
Table II. Airway insertion characteristics, oropharyngeal leak pressure and ventilatory parameters of the supraglottic airway devices (SADs) $(n=150)$.

\begin{tabular}{|c|c|c|c|c|}
\hline \multirow[t]{2}{*}{ Parameter } & \multicolumn{3}{|c|}{ No. (\%) } & \multirow[t]{2}{*}{ p-value } \\
\hline & i-gel $(n=50)$ & Supreme $(n=50)$ & ProSeal $(n=50)$ & \\
\hline SAD size & & & & $<0.001$ \\
\hline 3 & $24(48)$ & $10(20)$ & $6(12)$ & \\
\hline 4 & $26(52)$ & $21(42)$ & $33(66)$ & \\
\hline 5 & 0 & $19(38)$ & $11(22)$ & \\
\hline Ease of SAD insertion & & & & 0.105 \\
\hline 1st attempt & $44 / 49(90)$ & $41(82)$ & $36(72)$ & \\
\hline 2nd attempt & $3 / 49(6)$ & $5(10)$ & $7(14)$ & \\
\hline 3rd attempt & 0 & $1(2)$ & $2(4)$ & \\
\hline Intubated & $2 / 49(4)$ & 0 & 0 & \\
\hline Size change required & 0 & $3(6)$ & $5(10)$ & \\
\hline Duration of SAD insertion* (s) & $23.58(12-35)$ & $25.10(16-60)$ & $26.34(15-60)$ & 0.477 \\
\hline Ventilation mode & & & & 0.590 \\
\hline IPPV & 0 & 0 & 0 & \\
\hline Spontaneous & $4 / 47(9)$ & $2(4)$ & $2(4)$ & \\
\hline Both & $43 / 47(91)$ & $48(96)$ & $48(96)$ & \\
\hline Ease of gastric tube insertion & & & & 0.100 \\
\hline 1st attempt & $44 / 47(94)$ & $50(100)$ & $47(94)$ & \\
\hline 2nd attempt & $3 / 47(6)$ & 0 & $1(2)$ & \\
\hline Impossible & $0 / 47(0)$ & 0 & $2(4)$ & \\
\hline Gastric aspiration & & & & 0.415 \\
\hline Yes & $33 / 47(70)$ & $29(58)$ & $30(60)$ & \\
\hline No & $14 / 47(30)$ & $21(42)$ & $20(40)$ & \\
\hline Gastric aspiration volume $(\mathrm{mL})$ & $4.84(0-20)$ & $1.52(0-10)$ & $1.98(0-15)$ & $0.035^{\ddagger}$ \\
\hline Oropharyngeal leak pressure ${ }^{+}\left(\mathrm{cmH}_{2} \mathrm{O}\right)$ & $27.31 \pm 0.92$ & $23.60 \pm 0.70$ & $24.44 \pm 0.70$ & $0.003^{\ddagger}$ \\
\hline
\end{tabular}

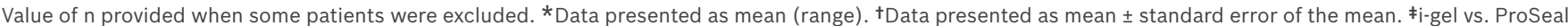
and Supreme groups. IPPV: intermittent positive pressure ventilation

Table III. Intraoperative and postoperative complications of each group $(n=150)$.

\begin{tabular}{|c|c|c|c|c|}
\hline \multirow[t]{2}{*}{ Complication } & \multicolumn{3}{|c|}{ No. (\%) } & \multirow[t]{2}{*}{ p-value } \\
\hline & $\begin{array}{c}\text { i-gel } \\
(n=50)\end{array}$ & $\begin{array}{l}\text { Supreme } \\
(n=50)\end{array}$ & $\begin{array}{l}\text { ProSeal } \\
(n=50)\end{array}$ & \\
\hline \multicolumn{5}{|l|}{ Intraoperative } \\
\hline Air leak at induction & $3(6)$ & 0 & 0 & 0.047 \\
\hline Laryngospasm & 0 & $1(2)$ & 0 & 0.365 \\
\hline $\begin{array}{l}\text { Obstruction after } \\
\text { insertion }\end{array}$ & 0 & 0 & $1(2)$ & 0.365 \\
\hline Blood staining & $0 / 49(0)$ & $9(18)$ & $9(18)$ & 0.007 \\
\hline Gastric content staining & $1 / 49(2)$ & $1(2)$ & 0 & 0.599 \\
\hline \multicolumn{5}{|l|}{ Postoperative } \\
\hline \multicolumn{5}{|l|}{$\begin{array}{l}\text { Immediate } \\
\text { (in recovery area) }\end{array}$} \\
\hline Dysphagia & $0 / 49(0)$ & $7(14)$ & $3(6)$ & 0.020 \\
\hline Hoarseness & $0 / 49(0)$ & $1(2)$ & $2(4)$ & 0.367 \\
\hline Sore throat & $2 / 49(4)$ & $14(28)$ & $15(30)$ & 0.002 \\
\hline \multicolumn{5}{|l|}{$\begin{array}{l}\text { Persistent } \\
\text { (the following day) }\end{array}$} \\
\hline Dysphagia & $0 / 49(0)$ & $2(4)$ & $1(2)$ & 0.367 \\
\hline Hoarseness & $0 / 49(0)$ & 0 & $1(2)$ & 0.369 \\
\hline Sore throat & $2 / 49(4)$ & $8(16)$ & $4(8)$ & 0.116 \\
\hline
\end{tabular}

Value of $\mathrm{n}$ provided when some patients were excluded. of safer controlled ventilation at higher airway pressures if required. ${ }^{(10)}$ Our finding was in contrast to those of previous studies comparing all three devices. Van Zundert et al evaluated the three devices in 150 patients and demonstrated that the mean leak pressure of the i-gel, measured immediately after insertion, was the lowest $\left(30 \mathrm{cmH}_{2} \mathrm{O}\right)$ among the three airway devices. ${ }^{(8)}$ Likewise, Mukadder et al, who also studied the three devices in 150 patients, similarly showed that the initial leak pressure was lower in the i-gel $\left(21 \mathrm{cmH}_{2} \mathrm{O}\right)$ group; however, the leak pressures of the Supreme and ProSeal groups were $24.90 \mathrm{cmH}_{2} \mathrm{O}$ and $23.90 \mathrm{cmH}_{2} \mathrm{O}$, respectively, which were similar to the leak pressures recorded in our study..$^{(9)}$

A possible reason for the i-gel's higher leak pressure in our study, as compared with previous studies, is the modification applied to its weight-based size selection criteria to account for the 10-kg overlap between sizes 3 and 4 . Nevertheless, there was a higher incidence of significant air leakage in the i-gel group, with three cases of the size $3 \mathrm{i}$-gel as opposed to none in the other devices. This could be due to the cuffless and gel-like nature of the i-gel, which makes it more prone to air leaks if the anatomical fit is insufficient for oesophageal and pharyngeal seals. Interestingly, a recent meta-analysis comparing the i-gel and ProSeal SADs demonstrated that when a neuromuscular 
blocker was used, the leak pressure of the ProSeal was higher than that of the i-gel SAD. However, there was no statistical difference in leak pressure when a neuromuscular blocker was not used. ${ }^{(11)}$ Our study did not use neuromuscular blockers during anaesthetic induction, as the types of surgical cases in our study did not warrant their use for muscle relaxation or surgical access. All our patients were either spontaneously breathing from the outset or resumed spontaneous ventilation after a short period of controlled ventilation. Notably, there is some evidence in the literature that spontaneous ventilation can allow a malpositioned LMA to remain undetected until a check is conducted with controlled ventilation, and that the folded epiglottis is more likely to have high airway pressure and leakage with controlled than spontaneous ventilation. ${ }^{(12,13)}$

We found that the i-gel was easier to successfully insert at the first attempt. It was also associated with shorter effective airway time than the ProSeal and Supreme SADs (Table II), although the differences demonstrated were not statistically significant due to limited power. Mukadder et al encountered no difference among the devices in terms of insertion success, although the insertion time was found to be shorter with i-gel..$^{(9)}$ Similarly, Teoh et al's trial of both the i-gel and Supreme SADs demonstrated comparable ease and duration of insertion between the two tested devices. ${ }^{(14)}$ A meta-analysis comparing the i-gel and Supreme SADs also concluded that there was no statistical difference in device placement time and first attempt insertion success rates. ${ }^{(15)}$ Nevertheless, other researchers have previously highlighted the i-gel's higher first attempt success rates and its shorter insertion time as compared with the ProSeal SAD. ${ }^{(16-19)}$ We postulated that the i-gel SAD should be easier to insert due to its unique gel-like material, shape and contour, buccal stabiliser, and epiglottis blocker that minimises epiglottis downfolding. The faster effective airway time of the i-gel SAD can be explained by the cuffless nature of the device, which obviates the necessity to inflate the cuff during insertion. Conversely, Van Zundert et al showed in their study that the Supreme SAD was easier to insert and had a shorter effective airway time than the ProSeal and i-gel SADs. ${ }^{(8)}$ Other studies also demonstrated that the Supreme was better in terms of first attempt success rates. ${ }^{(20,21)}$ The difference in the findings could be attributed to the SAD insertion techniques we used as compared with the other studies, e.g. Van Zundert et al employed the laryngoscope- and gastric tube-guided SAD insertion techniques in their study. ${ }^{(8)}$

The gastric tubes were inserted successfully at the first attempt in all patients in the Supreme group, and with a $94 \%$ success rate in both the i-gel and ProSeal groups. In 2 (4\%) patients in the ProSeal group, gastric tube passage was impossible. The insertion of gastric tubes in the i-gel group was slightly more difficult than in the Supreme group despite the use of a smaller 12 FG tube, due to the narrower gastric port. Mukadder et al showed in their study that gastric tube insertion had a similar success rate for the i-gel and Supreme groups, but was more difficult with the ProSeal group..$^{(9)}$ Teoh et al demonstrated no difference in the success rate of gastric tube insertion for the Supreme and i-gel groups. ${ }^{(12)} \mathrm{A}$ number of other studies reported gastric tube insertion failure in patients who used ProSeal, although it has not been reported in i-gel patients. ${ }^{(16,17,22)}$

Less airway morbidity and fewer complications were observed in the i-gel group as compared with the other two devices in our study. Blood staining of the airway device, which could be indicative of airway mucosal trauma, was observed in the ProSeal and Supreme groups, but not in the i-gel group. Furthermore, postoperative complaints of sore throat, voice hoarseness and dysphagia were either less evident or not observed in the latter group. Similar to our findings, the i-gel group in Mukadder et al's study had fewer reports of blood staining and other postoperative complications. ${ }^{(9)}$ These findings have been demonstrated by other studies as well. ${ }^{(23,24)}$ The i-gel SAD has a non-inflatable cuff that was designed to provide an anatomical fit over the perilaryngeal structures, minimising the risk of compression of neurovascular structures in these tissues and thereby reducing the incidence of airway complications. ${ }^{(25,26)}$

Our study had several limitations. Firstly, it was unblinded, as the investigators could not be blinded during airway management, hence presenting the possibility of researcher bias. Secondly, the oropharyngeal leak pressure was only measured once, at the start of the procedure, although the leak pressure may change over time. The reason for not measuring leak pressure repeatedly was that the unparalysed patients would only be ventilated for a short period of time before resuming spontaneous breathing, hence only the initial leak pressure was deemed important. In contrast to other similar studies, we did not perform bronchoscopy through the airway device to visualise the laryngeal image. We felt that it was logistically difficult and time-consuming to perform bronchoscopy for all patients; it was also not representative of the daily practice of our unit, given its high caseload and patient turnover. For the purpose of our study, SADs were not reinserted following failed gastric tube insertion if there were no ventilation issues. However, it must be noted that a failure to insert the gastric tube might indicate an incorrectly placed SAD, which might affect the oropharyngeal leak pressure.

In conclusion, our study demonstrated that the ProSeal, Supreme and i-gel SADs can provide a safe airway. We also showed that although the three devices were comparable in terms of ease and duration of placement, the i-gel SAD produced superior results in initial oropharyngeal leak pressure and airway morbidity compared with the ProSeal and Supreme SADs.

\section{REFERENCES}

1. Brain Al. The laryngeal mask--a new concept in airway management. $\mathrm{Br}$ J Anaesth 1983; 55:801-5.

2. Barker P, Langton JA, Murphy PJ, Rowbotham DJ. Regurgitation of gastric contents during general anaesthesia using laryngeal mask airway. $\mathrm{Br}$ J Anaesth 1992; 69:314-5.

3. Polat R, Aydin GB, Ergil J, et al. [Comparison of the i-gel ${ }^{\mathrm{TM}}$ and the Laryngeal Mask Airway Classic ${ }^{\mathrm{TM}}$ in terms of clinical performance]. Rev Bras Anestesiol 2015; 65:343-8. Portuguese.

4. Bamgbade OA, Macnab WR, Khalaf WM. Evaluation of the i-gel airway in 300 patients. Eur J Anaesthesiol 2008; 25:865-6.

5. Gatward JJ, Cook TM, Seller C, et al. Evaluation of the size 4 i-gel airway in one hundred non-paralysed patients. Anaesthesia 2008; 63:1124-30.

6. Schmidbauer W, Bercker S, Volk T, et al. Oesophageal seal of the novel 
supralaryngeal airway device I-Gel in comparison with the laryngeal mask airways Classic and ProSeal using a cadaver model. Br J Anaesth 2009; 102:135-9.

7. Eschertzhuber S, Brimacombe J, Hohlrieder M, Keller C. The laryngeal mask airway Supreme--a single use laryngeal mask airway with an oesophageal vent. A randomised, cross-over study with the laryngeal mask airway ProSeal in paralysed, anaesthetised patients. Anaesthesia 2009; 64:79-83.

8. Van Zundert TC, Brimacombe JR. Similar oropharyngeal leak pressures during anaesthesia with i-gel, LMA-ProSeal and LMA-Supreme Laryngeal Masks. Acta Anaesthesiol Belg 2012; 63:35-41.

9. Mukadder S, Zekine B, Erdogan KG, et al. Comparison of the proseal, supreme, and i-gel SAD in gynecological laparoscopic surgeries. ScientificWorldJournal 2015; 2015:634320.

10. Cook T, Howes B. Supraglottic airway devices: recent advances. Contin Educ Anaesth Crit Care Pain 2011; 11:56-61.

11. Park SK, Choi GJ, Choi YS, Ahn EJ, Kang H. Comparison of the i-gel and the laryngeal mask airway proseal during general anesthesia: a systematic review and meta-analysis. PLoS One 2015; 10:e0119469.

12. Moustafa MA, Abdelhady MM. Fiberoptic assessment of the Laryngeal Mask Airway (Laryseal) position after one hour of positive pressure ventilation: an observational study. J Clin Anesth 2014; 26:480-4.

13. Sharma B, Sood J, Sahai C, Kumra VP. Troubleshooting ProSeal LMA. Indian J Anaesth 2009; 53:414-24.

14. Teoh WH, Lee KM, Suhitharan T, et al. Comparison of the LMA Supreme vs the i-gel in paralysed patients undergoing gynaecological laparoscopic surgery with controlled ventilation. Anaesthesia 2010; 65:1173-9.

15. Chen X, Jiao J, Cong X, Liu L, Wu X. A comparison of the performance of the I-gel ${ }^{\mathrm{TM}}$ vs. the LMA-S ${ }^{\mathrm{TM}}$ during anesthesia: a meta-analysis of randomized controlled trials. PLoS One 2013; 8:e71910.

16. Hayashi K, Suzuki A, Kunisawa T, et al. [A comparison of the single-use i-gel with the reusable laryngeal mask airway Proseal in anesthetized adult patients in Japanese population]. Masui 2013; 62:134-9. Japanese.

17. Chauhan G, Nayar P, Seth A, et al. Comparison of clinical performance of the I-gel with LMA proseal. J Anaesthesiol Clin Pharmacol 2013; 29:56-60.

18. Jeon WJ, Cho SY, Baek SJ, Kim KH. Comparison of the Proseal LMA and intersurgical I-gel during gynecological laparoscopy. Korean J Anesthesiol 2012; 63:510-4.

19. Sharma B, Sehgal R, Sahai C, Sood J. PLMA vs. I-gel: A Comparative Evaluation of Respiratory Mechanics in Laparoscopic Cholecystectomy. J Anaesthesiol Clin Pharmacol 2010; 26:451-7.

20. Chew EE, Hashim NH, Wang CY. Randomised comparison of the LMA Supreme with the I-Gel in spontaneously breathing anaesthetised adult patients. Anaesth Intensive Care 2010; 38:1018-22.

21. Seet E, Rajeev S, Firoz T, et al. Safety and efficacy of laryngeal mask airway Supreme versus laryngeal mask airway ProSeal: a randomized controlled trial. Eur J Anaesthesiol 2010; 27:602-7.

22. Singh I, Gupta M, Tandon M. Comparison of Clinical Performance of I-Gel with LMA-Proseal in Elective Surgeries. Indian J Anaesth 2009; 53:302-5.

23. Das A, Majumdar S, Mukherjee A, et al. i-gel ${ }^{\mathrm{TM}}$ in Ambulatory Surgery: A Comparison with LMA-ProSeal ${ }^{\mathrm{TM}}$ in Paralyzed Anaesthetized Patients. J Clin Diagn Res 2014; 8:80-4.

24. Ragazzi R, Finessi L, Farinelli I, Alvisi R, Volta CA. LMA Supreme ${ }^{\mathrm{TM}}$ vs i-gel ${ }^{\mathrm{TM}}$--a comparison of insertion success in novices. Anaesthesia 2012; 67:384-8.

25. Levitan RM, Kinkle WC. Initial anatomic investigations of the I-gel airway: a novel supraglottic airway without inflatable cuff. Anaesthesia 2005; 60:1022-6.

26. Helmy AM, Atef HM, El-Taher EM, Henidak AM. Comparative study between I-gel, a new supraglottic airway device, and classical laryngeal mask airway in anesthetized spontaneously ventilated patients. Saudi J Anaesth 2010; 4:131-6. 